\title{
Just a sore throat? Uncommon causes of significant respiratory disease
}

\author{
Dalia Wahab, ${ }^{1}$ Julia Bichard, ${ }^{2}$ Anand Shah, ${ }^{1}$ Bhupinder Mann ${ }^{3}$
}

${ }^{1}$ Department of Respiratory, Royal Brompton Hospital, London, UK

${ }^{2}$ Palliative Care Department, Royal Free Hospital,

London, UK

${ }^{3}$ Department of Respiratory Medicine, West Middlesex University Hospital, Isleworth, Middlesex, UK

\section{Correspondence to}

Dr Dalia Wahab,

D.Wahab@rbht.nhs.uk

To cite: Wahab $D$ Bichard J, Shah A, et al. BMJ Case Rep Published online: [please include Day Month Year] doi:10.1136/ bcr-2013-008739

\section{SUMMARY}

We present two uncommon underlying causes of a sore throat which, if missed or delayed in diagnosis, can lead to disastrous consequences. Our first case is of Lemierre's syndrome diagnosed in a 21-year-old man presenting with a 5-day history of sore throat, fever, right-sided pleuritic chest pain and bilateral pulmonary nodules on CT imaging. Fusobacterium necrophorum cultured from peripheral blood and an occluded left internal jugular vein on ultrasound lead to an eventual diagnosis. Our second case presents a 29-year-old woman with a 5-day history of sore throat, fever and right-sided pleuritic chest pain. A left-sided quinsy was diagnosed and aspirated and the patient was discharged home. She represented shortly with worsening pleuritic pain and was found to have a right-sided pleural effusion with descending mediastinitis originating from the tonsillar abscess. Delayed diagnosis resulted in open thoracotomy, decortication and prolonged intravenous antibiotics.

\section{BACKGROUND}

Respiratory disease as a complication of ear, nose and throat (ENT) pathology is rare, but delay in diagnosis and subsequent definitive treatment has been found to be one of the principle factors leading to increased morbidity and mortality. We present two cases highlighting the importance of a thorough ENT history during the evaluation of a patient with respiratory disease to clinch a definitive diagnosis and, vice versa, a high index of suspicion of respiratory complications in patients presenting with ENT symptoms.

Lemierre's syndrome is a rare oropharyngeal infection, usually caused by Fusobacterium necrophorum and results in sepsis as well as spread of infected emboli from the adjacent infected internal jugular vein (IJV). It commonly occurs in men with a mean age of $16-25$ years. ${ }^{1-3}$ Over the past 15 years, its incidence has been increasing which could be due to a decrease in antibiotic use, better diagnostic facilities and more worryingly to antibiotic resistance. ${ }^{2}{ }^{4}$ On the other hand, parapharyngeal abscess is a common complication of acute tonsilitis which can spread and cause serious life threatening comlications, which are rare but if not diagnosed early and managed aggressively, they can lead to high morbidity and mortality with poor prognosis despite optimum current therapy.

\section{CASE PRESENTATION}

\section{First case}

A 21-year-old man presented to our hospital with a 5 -day history of fever and rigours. This was associated with a cough productive of green sputum, an initial sore throat that had resolved prior to presentation, mild dyspnoea on exertion and right-sided pleuritic chest pain. He had no significant medical history and was not on any regular medications. He worked as a roofer, and gave a history of moderate alcohol intake and a 2.5-pack-year smoking history. He had never used recreational drugs and had not travelled recently. On examination, he had fever at $38.1^{\circ} \mathrm{C}$, tachycardic at $110 \mathrm{bpm}$ with a blood pressure of $129 / 79$. His respiratory rate was 20 , with oxygen saturations of $97 \%$ on room air. His tonsillar examination revealed mild inflammation bilaterally but no pus was visible. There was no evidence of lymphadenopathy, and a full systemic examination was unremarkable. In particular, he had no signs of meningism, rash or neck erythema or swelling.

\section{Second case}

A 29-year-old woman presented to accident and emergency department with a 5-day history of sore throat and fever. She also reported a minor rightsided pleuritic chest pain on the day of admission but no dyspnoea. Two days prior to admission, she was started on penicillin $\mathrm{V}$ by her general practitioner. She had no medical history and had never smoked. On examination, she had fever at $38.3^{\circ} \mathrm{C}$ and tachycardic at $118 \mathrm{bpm}$. She had a prominent left tonsillar abscess and mild dullness to percussion at the right lung base.

\section{INVESTIGATIONS}

\section{First case}

Blood tests revealed a normal white cell count but a $C$ reactive protein was raised at $414 \mathrm{mg} / \mathrm{l}$. A chest radiograph revealed normal lung fields with a normal cardiac size and contour. Throat swabs, sputum and urine cultures showed no growth. On the second day, Gram-negative rods were detected in blood cultures and antibiotics were changed accordingly. The following day, it was noted on chest examination that there was dullness to percussion in the right-lower zone and the patient developed worsening hypoxia (oxygen saturations 93\% on room air). Repeat chest radiography revealed a small right-sided pleural effusion. His white cell count had risen to $25 \times 109 / 1$ and a $\mathrm{C}$ reactive protein was persistently elevated $(292 \mathrm{mg} / \mathrm{l})$. For this reason, a CT scan of his chest and abdomen was performed which showed small bilateral pleural effusions, along with multiple pulmonary nodules ranging in size from a few millimetres to $2.6 \mathrm{~cm}$ in all lobes but with a basal predominance. Some of the larger nodules contained air suggesting 

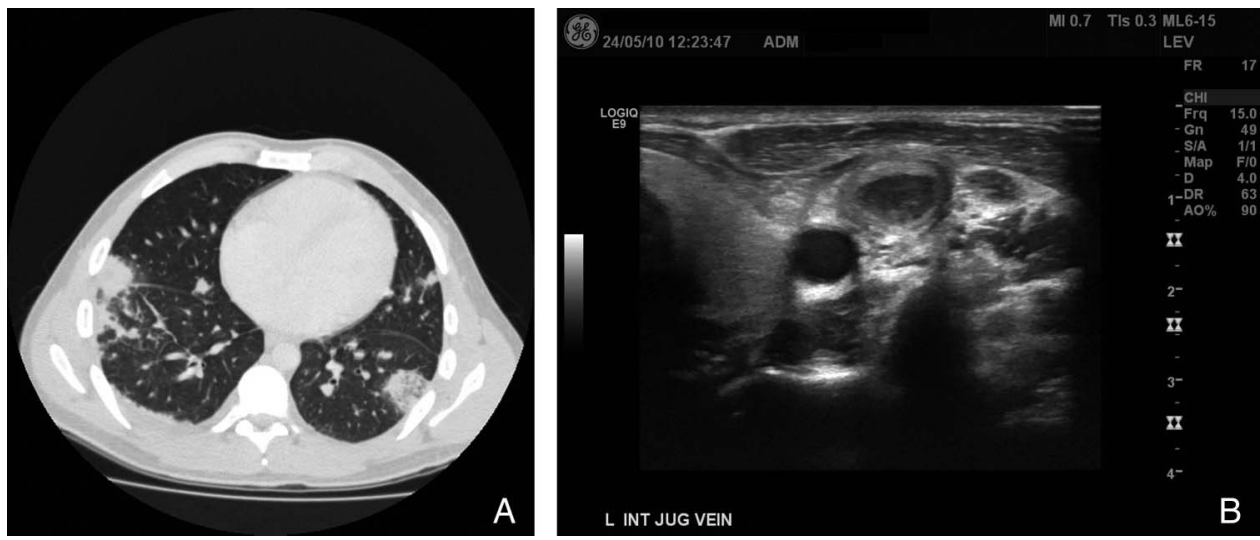

Figure 1 (A) CT of the chest showing bilateral multiple pulmonary nodules with small bilateral effusions. (B) Ultrasound of the left internal Jugular vein detected complete thrombosis within the jugular vein.

cavitation. There was additional bibasal atelectasis and consolidation. The liver, spleen, kidneys and pancreas were normal, and there were no destructive bony lesions. A vasculitic screen was normal and there were no stigmata of bacterial endocarditis. Atypical serology and respiratory viral screen were all normal. The Gram-negative rod in his blood culture was identified as an anaerobe and on day 7, F necrophorum was identified. Based on this result, an ultrasound scan of the patient's neck was performed and this showed that the left IJV was completely occluded with thrombus, in keeping with a diagnosis of Lemierre's syndrome (figure 1).

\section{Second case}

The initial blood tests revealed a white cell count of $15 \times 109 / 1$, and $C$ reactive protein of $460 \mathrm{mg} / \mathrm{l}$. Initial chest radiograph on admission showed a slight blunting of the right costophrenic angle. Blood cultures taken on admission revealed no growth. She was initially admitted under the ENT specialists who aspirated $2 \mathrm{ml}$ of pus from the left tonsillar abscess and was managed with $24 \mathrm{~h}$ of intravenous antibiotics prior to discharge with a 7-day course of oral metronidazole and clarithromycin following minor improvement in symptoms. The clinical and radiological evidence of a small right pleural effusion was not actively treated by the admitting team. Three days later she represented to the accident and emergency department with dyspnoea, worsening right-sided pleuritic chest pain, a white cell count of $20 \times 109 / 1$, and a C reactive protein of $111 \mathrm{mg} / \mathrm{l}$. A repeat chest radiograph revealed a loculated right-sided pleural effusion extending into the horizontal fissure. A CT scan of her neck and chest showed complete collapse of the rightlower lobe with a moderately sized loculated right pleural effusion with pleural enhancement, and a small left-sided pleural effusion with basal atelectasis. There was an additional fluid collection in the mediastinum posterior to the oesophagus, extending from the gastro-oesophageal junction to the level of the hyoid/C5 vertebra, extending proximally and containing blebs of air. Ultrasound scan of the pleural effusion revealed a complex multiseptated effusion with no dominant locule. A radiologically guided 12 -french Seldinger chest drain was inserted but had little output. Pleural fluid culture did not reveal any growth and acid-fast bacilli smear and culture were negative (figure 2).

\section{TREATMENT}

\section{First case}

In view of his clinical presentation, he was initially started on intravenous benzylpenicillin $1.2 \mathrm{~g}$ four times a day and clarithromycin $500 \mathrm{mg}$ twice a day. On the second day Gram-negative rods were identified on blood cultures and hence his antibiotics were changed to intravenous tazocin $4.5 \mathrm{~g}$ three times a day as
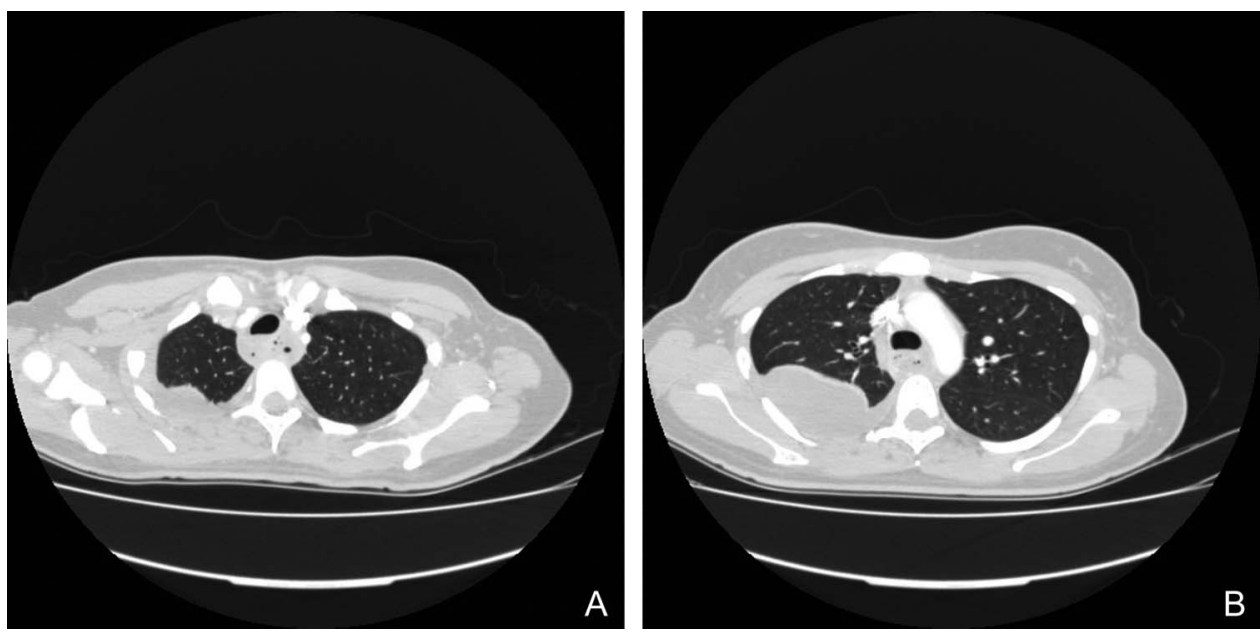

Figure 2 (A) CT of the upper chest showing loculated fluid collection extending from the right-upper zone. (B) CT of the chest showing the fluid collection extending further downwards. 
per microbiology team advice in view of ongoing fever. The organism was then further identified as an anaerobe, and intravenous metronidazole $400 \mathrm{mg}$ three times a day was added with improvement of his temperature and inflammatory markers. On diagnosis of Lemierre's syndrome, the patient was treated with a total of 3 weeks of tazocin $4.5 \mathrm{~g}$ three times a day and metronidazole $400 \mathrm{mg}$ three times a day intravenously, followed by oral metronidazole for 3 weeks. He was also anticoagulated for 3 months with warfarin for the management of the left IJV thrombosis.

\section{Second case}

The patient was initially admitted under the ENT specialists who following diagnosis of a left-sided tonsillar abscess performed a tonsillar aspiration of $2 \mathrm{ml}$ of pus. She was subsequently treated with $24 \mathrm{~h}$ of intravenous benzylpenicillin $1.2 \mathrm{~g}$ four times a day and metronidazole $400 \mathrm{mg}$ three times a day before being discharged on a 7-day course of oral metronidazole $500 \mathrm{mg}$ three times a day and clarithromycin $500 \mathrm{mg}$ twice a day following minor improvement in symptoms There was inadequate focus on the patient's respiratory symptoms and evidence of a small right pleural effusion was overlooked.

On re-admission with right-sided pleuritic chest pain with identification of an increased loculated pleural effusion on chest radiograph, the patient was started on tazocin $4.5 \mathrm{~g}$ three times a day and intravenous metronidazole $400 \mathrm{mg}$ three times a day and a Seldinger chest drain was inserted for attempted drainage. Following the CT findings which revealed a multiseptated loculated pleural effusion and a posterior mediastinal collection indicating a likely descending necrotising mediastinitis the patient was immediately transferred under the joint care of the cardiothoracic and upper gastrointestinal surgical teams at a tertiary referral centre for a thoracotomy and washout of the right thoracic and mediastinal collections. Despite initial video-assisted thorascopic right-sided pleural drainage the patient had ongoing evidence of residual infection with ongoing fever, raised inflammatory markers and radiographic evidence of residual loculated pleural fluid. She therefore underwent further right-sided thoractomy with decortication and was eventually discharged 6 weeks after admission with a further 6 -week course of intravenous antibiotics with ertapenem $1 \mathrm{~g}$ once a day via a peripherally inserted central catheter (PICC line) and oral fluconazole $400 \mathrm{mg}$ once a day.

\section{OUTCOME AND FOLLOW-UP}

\section{First case}

Upon discharge he was well and inflammatory markers had normalised.

\section{Second case}

Following this protracted admission and prolonged antibiotic course the patient made a good recovery but is still undergoing physiotherapy to return to baseline function. The development of a right-sided complex empyema requiring eventual decortication due to initial delayed diagnosis of descending necrotising mediastinitis with suboptimal treatment with oral antibiotics will likely have some restriction on the patient's long-term lung function.

\section{DISCUSSION}

\section{First case}

Lemierre's syndrome is a rare oropharyngeal infection which results in sepsis and spread of infected emboli from the adjacent
IJV. It occurs over a number of stages ${ }^{6}$ : the initial stage is infection of the primary site which is commonly the oropharynx (the palatine tonsil in $87 \%$ of cases). ${ }^{1}$ Other possible primary sites include: otitis media, mastoiditis, sinusitis and parotitis. ${ }^{7}$ Following this first stage, the infection spreads into the lateral pharyngeal space, usually via the lymphatic system, into the IJV where it results in thrombophlebitis with thrombus formation and septicaemia ${ }^{1}$ as for our patient. At this stage, neck pain and tenderness can occur, but only in 53\% of cases. ${ }^{1}$ Indeed in our case there were no neck symptoms or signs. Infected thrombus that has formed at the IJV can result in embolisation causing infection elsewhere most commonly in the lung in $92 \%$ of cases. ${ }^{17}$ This can result in various complications including pulmonary infiltrates, pleural effusion, lung abscess and empyema. ${ }^{2}$ Other possible sites where infection can spread include: joints (17\% of cases $\left.{ }^{7}\right)$, bone, liver, spleen, skin, soft tissue, kidneys, brain parenchyma and meninges. ${ }^{2} 68$ The whole process usually takes around 7 days. In our case, the presence of bilateral, bilobar infiltrates on the chest x-ray and CT suggested the presence of septic emboli.

Lemierre's syndrome usually occurs in young adults with a mean age of $16-25$ years, ${ }^{3}$ more commonly in men $(75 \%$ of cases). ${ }^{1}{ }^{2}$ It is usually caused by a Gram-negative, anaerobic bacillus: $F$ necrophorum in $87 \%$ of cases. ${ }^{1}$ In our case, it was the discovery of this bacterium which triggered the suspicion of Lemierre's syndrome, highlighting the importance of taking blood cultures prior to antibiotic start, in order not to cloud diagnosis. F necrophorum is part of normal flora of the oral cavity, female genitalia and gastrointestinal tract. ${ }^{9}$ Other microorganisms that can be associated with Lemierre's syndrome include Bacteroids spp, Peptostreptococcus spp, Streptococcus spp, Staphylococcus aureus and Eikenella corrodens. ${ }^{4} 6910$

Lemierre's syndrome was initially reported in 1900 where a case was reviewed by Courmont and Cade. ${ }^{4}$ In 1936 Andre Lemierre formally described 20 French cases with this syndrome. $^{8} 11$ This has an incidence of about 1 per million per year, ${ }^{11} 12$ however this varied over time, being initially common during the 20th century, before the use of antibiotics. Following antibiotic discovery, the incidence has decreased over three decades with only 40 cases identified between 1950 and 1995 with only two cases between 1980 and 1990s) as was found by Leuger and Clover. ${ }^{9}$ This has led to Lemierre's being termed: 'the forgotten disease'. ${ }^{13}$ However, over the past 15 years, the incidence has been increasing and it has been suggested this may be secondary to decrease in antibiotic use, to better diagnostic facilities and more worryingly to antibiotic resistance. ${ }^{2} 413$ Death rate preantibiotics time was $90 \% .^{9}{ }^{12}$ This has now deceased to $8-15 \%$ with antibiotics. ${ }^{10}$

Treatment is usually with a prolonged course of antibiotics of a minimum period of 6 weeks. ${ }^{1}$ This includes an initial intravenous treatment for approximately 2 weeks or until clinical improvement, ${ }^{13}$ followed by oral antibiotics. Penicillin, clindamycin, metronidazole and chloramphenicol are commonly used, but most usually a combination of penicillin and metronidazole $^{2} 91013 \quad 14$ is given to provide wider anaerobic cover. For our case, a combination of intravenous tazocin and metronidazole for 3 weeks, followed by oral metronidazole for 3 weeks was used. Surgery and ligation of thrombosed vessels is now rarely required and only used where there is uncontrolled sepsis and septic emboli occurring despite medical therapy. ${ }^{8}$

Anticoagulation therapy is still controversial as there are no controlled, randomised trials available. ${ }^{8}{ }^{10}$ At present, it is mainly considered for retrograde extension of thrombus into the cavernous sinus or in cerebral infarction, ${ }^{1}{ }^{13}$ but there are 
conflicting views in the literature about whether anticoagulation may risk extension of the infection. ${ }^{2}$ On the other hand, some papers recommend the use of anticoagulation as it is thought to reduce the time to clearance of the infected thrombus, ${ }^{48}$ reduce septic foci and limit emboli shower, particularly if emboli persist despite antibiotic therapy. ${ }^{13}$ There has also been suggestion of an underlying tendency to thrombophilia due to a hypercoagulable state in Lemierre's syndrome and is thought that anticoagulation should be considered for this reason on an individual basis. ${ }^{15}$ If anticoagulation is considered it is usually recommended for a period of 3 months. ${ }^{8}$

\section{Second case}

Parapharyngeal abscess is a common complication of acute tonsillitis which usually affects young adults as for the patient presented but can occur at any age. ${ }^{5}$ Its incidence range between $9 \%$ and $16 \%$ which has decreased with the use of antibiotics and improved oral hygiene. ${ }^{16}$ However, it can cause serious lifethreatening complications with high morbidity and mortality which can develop despite treatment. ${ }^{17}$ For this reason, it requires early detection and aggressive management. If an abscess occurs, it requires adequate treatment with antibiotics and needle aspiration, incision and drainage or immediate tonsillectomy. ${ }^{5}$ If inadequately treated, the infection can spread downward through the anatomical planes inferiorly into the mediastinum, most commonly through the retrovisceral space and 'danger space'. ${ }^{18-20}$ The danger space is found between the alar and prevertebral fascia, and deep cervical fascia, extending from the skull base through posterior mediastinum down to the diaphragm. ${ }^{18}$ Common pathogens isolated in parapharyngeal abscess include: Streptococcus pyogenes (usually the predominant organism), $S$ aureus. Haemophilus influenzae as well as anaerobic organisms including Prevotella spp, Porphyromonas spp, Fusobacterium spp and Peptostreptococcus spp..$^{5} 18$ 20-23

The spread of peritonsillar abscess can cause serious complications particular in the immunosuppressed and patients with other comorbidities who are particularly at greater

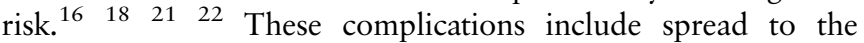
jugular vein causing septic thromboemboli, carotid artery pseudoaneurysm or rupture, laryngeal oedema leading to airway compromise, epidural involvement, meningitis and disseminated intravascular coagulation. The downward spread, as for our case, can also result in mediastinitis (including descending necrotising mediastinitis), pericarditis, pericardial effusion, pneumonia, empyema and ARDS. ${ }^{5} 16 \quad 1822 \quad 24$ These are lifethreatening complications which, if not detected and treated early, can have high death rate with poor prognosis despite current management. Some studies reported a death rate from parapharygeal abscess of up to $20 \%,{ }^{23}$ and in some studies, this ranged between $7.1 \%$ and $41.7 \% .^{22}$

Our case patient had a prolonged hospitalisation and ITU admission and recurrent need for surgical intervention as a result of this complication. This case highlights the consequence of an inadequate focus on respiratory symptoms in a patient presenting with an ENT diagnosis alongside the consequence when a paratonsillar abscess in inadequately managed with suboptimal antibiotic therapy despite needle aspiration. Previous studies have suggested the need for surgical intervention with tonsillectomy within $48 \mathrm{~h}$ if there is no clinical improvement following needle aspiration and intravenous antibiotics alone in order to avoid such complications. ${ }^{25}$ Other studies have suggested the routine use of CT imaging to aid in diagnosing and localising the presence of an abscess, the presence of further secondary complications, the extent of such complications and assisting in image guidance for aspiration or surgical intervention. 1718

With a higher initial suspicion for and a better awareness of the importance of respiratory complications of paratonsillar abscess' this would have led to improved initial management and likely reduced morbidity and hospitalisation in this case.

\section{Learning points}

- Our two cases highlight the role of ear, nose and throat (ENT) pathologies as a potential source for significant severe respiratory disease.

- Although uncommon, initial signs of respiratory disease may be subtle and in the case of Lemierre's syndrome the ENT aetiology might be difficult to establish.

- A thorough history and a high index of suspicion are necessary to confirm the diagnosis, with early detection key to preventing serious mortality and morbidity.

Acknowledgements We would like to thank Daniel Edwin (IT Department at West Middlesex University hospital) for his contribution in obtaining the images for the two cases.

Contributors DW contributed in writing the background information, discussion, references for both cases, obtained consent from both patients as well as contributed in finalising the final version. JB contributed in writing the two case histories (presentation, investigations, treatment and follow-up). AS has clarified adjusted and contributed in writing the two cases including background information, case histories and discussion and in finalising the case reports. BM is the overall supervisor of the cases, contributed in guidance as well as in obtaining the images for the cases. He initiated the idea of choosing those two cases for submission.

\section{Competing interests None.}

Patient consent Obtained.

Provenance and peer review Not commissioned; externally peer reviewed.

\section{REFERENCES}

1 Dirks J, Bowie D. Sore throat progressing to embolic sepsis: a case of Lemierre's syndrome. Can Respir J 2010;17:e20-2.

2 Kristensen L Hagelskjaer, Prag J. Human necrobacillosis, with emphasis on Lemierre's syndrome. Clin Infect Dis 2000;31:524-32.

3 Kadhiravan T, Piramanayagam P, Banga A, et al. Lemierre's syndrome due to community-acquired meticillin-resistant Staphylococcus aureus infection and presenting with orbital cellulitis: a case report. J Med Case Rep 2008;2:374.

4 Riordan T. Human infection with Fusobacterium necrophorum (Necrobacillosis), with a focus on Lemierre's syndrome. Clin Microbiol Rev 2007;20:622-59.

5 Galioto NJ. Peritonsillar abscess. Am Fam Physician 2008;77:199-202.

6 Bukari SM, Roxas R, Kamat D. Variant of Lemierre's syndrome secondary to trauma. Int J Pediatr 2010;2010:123943.

7 Crum-Cianflone N, Mayer R. An unusual case of Lemierre's syndrome presenting as pyomyositis. Am J Med Sci 2008;335:499-501.

8 Alherabi A. A case of Lemierre syndrome. Ann Saudi Med 2009;29:58-60.

9 Ramirez S, Hild TG, Rudolph CN, et al. Increased diagnosis of Lemierre syndrome and other Fusobacterium necrophorum infections at a Children's Hospital. Pediatrics 2003; $112: \mathrm{e} 380$

10 Seo YT, Kim MJ, Kim JH, et al. Lemierre syndrome: a case of postanginal sepsis. Korean J Intern Med 2007;22:211-14.

11 Takazono T, Izumikawa K, Tsurutani J, et al. Lemierre's syndrome followed by acute respiratory distress syndrome successfully rescued by antibiotics and hemoperfusion with polymyxin B-immobilized fiber. Jpn J Infect Dis 2009;62:133-6.

12 Harris $C M$, Johnikin $M$, Rhodes $H$, et al. Lemierre's syndrome resulting from streptococcal induced otitis media and mastoiditis: a case report. J Med Case Rep 2009:3:6658.

13 Li HY, Grubb M, Panda M, et al. A sore throat-potentially life-threatening? J Gen Intern Med 2009:24:872-5.

14 Kushawaha A, Popalzai M, El-Charabaty E, et al. Lemierre's syndrome, re-emergence of a forgotten disease: a case report. Cases J 2009:2:6397. 
15 Lakshminarayana PH, Woodske ME. A unique case of Lemierre syndrome associated with thrombophilia in an adult and the role of anticoagulation. Case Report Med 2010;2010:982494.

16 Lee JK, Kim HD, Lim SC. Predisposing factors of complicated deep neck infection: an analysis of 158 cases. Yonsei Med J 2007:48:55-62.

$17 \mathrm{Oh} \mathrm{JH}, \mathrm{Kim}$ Y, Kim CH. Parapharyngeal abscess: comprehensive management protocol. ORL J Otorhinolaryngol Relat Spec 2007; 69:37-42.

18 Alaani A, Griffiths H, Minhas SS, et al. Parapharyngeal abscess: diagnosis, complications and management in adults. Eur Arch Otorhinolaryngol 2005;262:345-50.

19 Estrera AS, Landay MJ, Grisham JM, et al. Descending necrotizing mediastinitis: surgical management. Surg Gynecol Obstet 1983;157:545-52.
20 Brook I. Microbiology and management of peritonsillar, retropharyngeal, and parapharyngeal abscesses. J Oral Maxillofac Surg 2004;62:1545-50.

21 Greinwald HJ Jr, Wilson JF, Haggerty PG. Peritonsillar abscess: an unlikely cause of necrotizing fasciitis. Ann Otol Rhinol Laryngol 1995;104:133-7.

22 Yang SW, Lee MH, Lee YS, et al. Analysis of life-threatening complications of deep neck abscess and the impact of empiric antibiotics. ORL J Otorhinolaryngol Relat Spec 2008;70:249-56.

23 DS, Stanley RE. Deep neck abscesses_changing trends. J Laryngol Otol 1994;108:138-43.

24 Sichel JY, Attal P, Hocwald E, et al. Redefining parapharyngeal space infections. Ann Otol Rhinol Laryngol 2006;115:117-23.

25 Nielsen TR, Clement F, Andreassen UK. Mediastinitis - a rare complication of a peritonsillar abscess. J Laryngol Otol 1996;110:175-6.

Copyright 2013 BMJ Publishing Group. All rights reserved. For permission to reuse any of this content visit http://group.bmj.com/group/rights-licensing/permissions.

BMJ Case Report Fellows may re-use this article for personal use and teaching without any further permission.

Become a Fellow of BMJ Case Reports today and you can:

- Submit as many cases as you like

- Enjoy fast sympathetic peer review and rapid publication of accepted articles

- Access all the published articles

- Re-use any of the published material for personal use and teaching without further permission

For information on Institutional Fellowships contact consortiasales@bmjgroup.com

Visit casereports.bmj.com for more articles like this and to become a Fellow 